\title{
Efficacy of Magnesium and Alpha Lipoic Acid Supplementation in Reducing Premature Uterine Contractions
}

\author{
Eligio Parente ${ }^{1}$, Giulia Colannino ${ }^{2}$, Pasquale Ferrara ${ }^{1}$ \\ ${ }^{1}$ Obstetric and Gynecological Center "Villa delle Querce", Naples, Italy \\ ${ }^{2}$ Clinic Center "Trusso", Naples, Italy \\ Email: "eligio.parente@libero.it
}

Received 12 April 2014; revised 9 May 2014; accepted 5 June 2014

Copyright (C) 2014 by authors and Scientific Research Publishing Inc.

This work is licensed under the Creative Commons Attribution International License (CC BY).

http://creativecommons.org/licenses/by/4.0/

(c) (i) Open Access

\section{Abstract}

Objective: Premature uterine contractions represent one of the major symptoms related to preterm labor. So far, primary prevention of preterm labor is based on the early identification of symptoms and on pharmacological treatments which are prone of several secondary effects. In this double-blinded, randomized, placebo-controlled trial, the efficacy of a supplementation of magnesium and alpha-lipoic acid has been evaluated. Methods: Three hundred pregnant women at 14 - 34 weeks of gestation were enrolled and randomly divided to receive a daily single tablet containing a supplement of magnesium and alpha-lipoic acid (DAV ${ }^{\circledR}$ LoLiPharmasrl, Rome-Italy) or placebo until delivery. The incidence of episodes of preterm uterine contraction, associated or not with pain, as well as maternal need of hospitalization was evaluated. Results: Magnesium and lipoic acid supplementation was effective to significantly reduce the incidence of preterm uterine contractions compared to placebo. In particular, $52 \%$ of women who received the supplementation reported no symptoms of preterm uterine contractions throughout pregnancy, and persistent episodes of uterine contractions were significantly reduced compared to placebo $(20 \%$ vs $60 \%$, respectively). Furthermore, only $20 \%$ of subjects who received the supplementation required hospitalization, while it has been necessary for $40 \%$ of women who received placebo. Conclusions: Our findings suggest that supplementation with magnesium and lipoic acid is effective in reducing the incidence of premature uterine contraction and related episodes of hospitalization, compared to placebo. Nevertheless, further studies based on larger cohorts of patients are necessary to confirm the efficacy of these preliminary results.

"Corresponding author.

How to cite this paper: Parente, E., Colannino, G. and Ferrara, P. (2014) Efficacy of Magnesium and Alpha Lipoic Acid Supplementation in Reducing Premature Uterine Contractions. Open Journal of Obstetrics and Gynecology, 4, 578-583. 


\section{Keywords}

\section{Magnesium, Alpha-Lipoic Acid, Preterm Labor, Tocolysis, Pregnancy}

\section{Introduction}

Increased uterine-contraction frequency has been considered to lead to preterm labor and delivery, so that strategies for early detection and suppression of contractions have been advocated to reduce the incidence of premature birth [1]. Primary prevention of preterm labor is characterized by the common aim of lowering its prevalence, by improving maternal health and by avoiding risk factors during pregnancy. On the other hand, the goal of secondary prevention is the early identification of possible risk factors for preterm labor and the opportune treatment.

Premature labor can be treated with tocolytic drugs for inhibition of uterine contractions and individualized therapy consists of the selection of the tocolytic agent that is the most effective for each patient, with minimal side effects, chosen among several agents such as calcium antagonists, oxytocin-receptor antagonists, inhibitors of prostaglandin synthesis, nitric oxide (NO) donors, betamimetics and magnesium sulfate [2] [3]. Most of these drugs are not avoided of important side effects [4] that should be taken into account when choosing the right agent.

Magnesium is an oligo-element that plays a crucial role in more than 200 different chemical reactions in the body. Until a few years ago, it was an abundant mineral in several foods. However, nowadays, food processing practices essentially stripped magnesium out of most diets, causing several people to become magnesium deficient. It has been shown that during periods of extreme physical (i.e. pregnancy) or psychological stress, magnesium is often extensively consumed by the body. Interestingly, some preclinical and clinical studies showed that magnesium may be useful in the treatment of depression mainly because of its potential to block the ion channel of the NMDA receptor preventing its excessive activation [5] [6]. Many other studies have shown that a deficiency in magnesium contributes not only to the development of psychiatric disorders but also to several other health problems, including pregnancy disturbances. The capability of magnesium to prolong preterm labor was already described more than 50 years ago [7]. Moreover, clinical evidence shows that women in preterm labor have significantly decreased serum magnesium levels [8] and present a reduced permeability to calcium and magnesium concerning the exchange with fetal membranes [9]. It has been demonstrated that, at a pharmacologic concentration ( $5 \mathrm{mmol} / \mathrm{L}$ ), magnesium sulfate inhibits contractile response in pregnant human myometrial strips by a pattern that is consistent with both extra and intracellular mechanisms [10]. However, up to date the mechanism responsible of its tocolytic action is still not completely characterized. One hypothesis is that magnesium competes with calcium for entry into the myometrial cells through voltage-gated channels. Another possible explanation is that, since magnesium enhances nitric oxide synthase (NOS) activity [11], a reduction of magnesium would cause a consequent reduction of NO in the myometrium and in the placenta. In vitro studies have demonstrated that magnesium inhibited extracellular calcium entry, intracellular calcium release, cytosolic calcium oscillations, and phasic contractions of myometrial smooth muscle [12]. Moreover, it has been demonstrated that magnesium sulfate inhibits phosphatidylinositol-4,5-bisphosphate-specific phospholipase C activity and subsequent calcium release in cultured myometrial cells by a direct effect on phospholipase C [13].

Although there are several drugs or compounds marketed as tocolytic agents, the identification of a clear first-line tocolytic agent is still lacking. Thus, more research on innovative therapies with better profiles in terms of both efficacy and safety is warranted.

Alpha lipoic acid (ALA) is an organosulfur compound existing in two enantiomeric forms. Only the (R)-(+)enantiomer exists in nature and it is an essential cofactor of mitochondrial enzymes complexes important for aerobic metabolism [14]. ALA is present in almost all foods but in a very limited amount. Moreover, it is covalently bound and not readily available from dietary sources. Both enantiomers are included in several over-thecounter supplements and have been used for several clinical purposes, generally blended as antioxidants. As an antioxidant compound, ALA behaves as a scavenger of oxygen free radicals; it chelates metals, promotes the regeneration of endogenous antioxidants such as vitamin $\mathrm{C}$ and $\mathrm{E}$ and exerts anti-inflammatory properties [15]. Thus, the possibility exists that ALA synergistically interacts with magnesium by reducing some of the main 
factors related to the risk of preterm delivery. The aim of the present study was to evaluate whether pregnant women treated with a combination of magnesium and ALA could experience a significant reduction in preterm uterus contractions. Moreover, we evaluated whether the association of ALA and magnesium could significantly reduce the need of hospitalization compared to the placebo group.

\section{Methods}

\subsection{Subjects}

This double-blinded, randomized, placebo-controlled trial included three hundred healthy pregnant women, enrolled in the Gynecology and Obstetrics Clinic, Casa di Cura Villa delle Querce, Naples, Italy from January 2012 to January 2013. Inclusion criteria were age between 23 - 41 years, 14 - 34 weeks of gestation, intact membranes, cervical dilation of $\leq 2 \mathrm{~cm}$, and the dating of pregnancy confirmed through first trimester ultrasound scanning.

Of the recruited women, 100 were nulliparous and 200 multiparous with history of spontaneous delivery or cesarean section, among which 50 women reported previous miscarriages or preterm delivery (before 35 weeks of gestation). Subjects were excluded if they had preexisting diabetes mellitus or systolic blood pressure $\geq 140$ $\mathrm{mm} \mathrm{Hg}$ at enrollment, if had any serious health condition likely to affect the prenatal or postnatal growth and development of their offspring, including cancer, lupus, hepatitis, HIV/AIDS, or a diagnosed alcohol or chemical dependency. Vaginal smears were evaluated at the enrolment and patients who resulted positive for bacterial vaginosis were excluded. Women were asked to fill in questionnaires focused on overall health status, lifestyle behavior, diet and use of spasmolytic or tocolytic drugs in previous pregnancies.

The subjects were randomly assigned into an intervention group and a placebo group on a 5:1 basis. Two hundred fifty women (80 nulliparous and 170 multiparous, among which 40 women reported previous abortions and/or preterm delivery) were assigned to receive a daily single tablet containing magnesium and ALA (DAV ${ }^{\circledR}$ LoLiPharmasrl, Italy: magnesium $225 \mathrm{mg}$, alpha lipoic acid $100 \mathrm{mg}$ and vitamin B6 $1.3 \mathrm{mg}$ ) and fifty women (20 nulliparous and 30 multiparous, among which 10 women reported previous abortions and/or preterm delivery) received a daily single placebo tablet. A 5:1 randomization was chosen due to ethical reason, because it was known that patients assigned to placebo would be receiving a daily treatment with no possibility of direct benefit.

Both the physicians and the women were blinded to the treatment. The demographic characteristics of the enrolled patients were analyzed and no significant difference between the randomized groups was reported.

Before entering the study, a written informed consent was obtained from all subjects. The study was conducted after the local ethical committee approval.

\subsection{Outcomes}

The primary outcomes considered were:

- sporadic episodes of preterm uterine contractions;

- persistent episodes of preterm uterine contractions associated with pain;

- maternal need for hospital admission for threatened preterm labor.

Definition of uterine contractions as "sporadic" or "persistent" was based on a self-evaluation performed by the patients throughout the pregnancy. Sporadic episodes never required any specific pharmacological treatment while persistent episodes associated with pain required appropriate therapy with antispastic/tocolytic agents but no need of hospitalization.

\subsection{Follow Up}

From enrollment, women were followed up every 4 weeks until delivery. At each visit, blood pressure was measured, ultrasound examination was performed and blood samples were collected in order to monitor fetal and mother's health status and to investigate the safety of the treatment. Pregnancies that worsened and needed hospitalization were excluded from the treatment protocol but were still followed until delivery. Every woman has been checked a week after the expected day of delivery from a physician not involved in the study. No specific adverse events referable to the administration of the treatment were reported. 


\subsection{Statistical Analysis}

Comparison of the two treatments was performed using the Fischer's exact test and P values less than 0.05 were considered statistically significant.

The analysis was conducted using GraphPad Prism software (GraphPad Software, Inc., La Jolla, USA).

\section{Results}

This clinical trial included 300 pregnant women: 250 women received a daily single tablet containing magnesium and ALA supplement (DAV ${ }^{\circledR}$ LoLiPharmasrl, Italy: magnesium $225 \mathrm{mg}$, alpha lipoic acid $100 \mathrm{mg}$ and vitamin B6 $1.3 \mathrm{mg}$ ); 50 women received a daily single placebo tablet.

Among the 250 pregnant women treated with magnesium and ALA supplement, 70 women (28\%) reported single episodes of uterine contractions at different gestational ages, with no need of hospitalization; 50 women (20\%) reported persistent uterine contractions associated with pain, and were therefore hospitalized to establish proper pharmacological therapy (antispasmodic and/or tocolytic agents); in 10 of them, tocolytic therapy was necessary only starting from 34 weeks of gestation. The remaining 130 women (52\%) did not show any symptom of preterm uterine contractions and did not need hospitalization throughout the course of pregnancy.

Among the 50 pregnant women assigned to the placebo group, 30 (60\%) required antispasmodic and/or tocolytic agents starting from week 17 of gestation; among them, 10 women delivered before week 35 . The remaining 20 women (40\%) treated with placebo required at least two episodes of hospitalization for intravenous pharmacotherapy throughout pregnancy. Changes in the primary outcomes are summarized in Table 1.

\section{Discussion}

The results of this study clearly show that supplementation with magnesium and ALA from week 14 of gestation was effective in reducing the incidence of premature uterine contractions and related episodes of hospitalization. Thus, among the women taking daily magnesium and ALA supplement, 52\% reported no symptoms of preterm uterine contractions throughout pregnancy, while 28\% reported sporadic episodes of uterine contractions that spontaneously resolved with no need of pharmacotherapy or hospitalization. Conversely, among the women assigned to the placebo group, $60 \%$ needed antispasmodic and/or tocolytic therapy starting from week 17 of gestation, and $40 \%$ needed hospital admission at least twice throughout pregnancy.

Previous studies have evaluated the efficacy of magnesium in reducing uterine contractions both in vivo and in vitro. Thus, a recent study has evaluated the efficacy of magnesium sulfate infusion for 48 hours as a second-line tocolytic therapy in women at 22 to 34 weeks of gestation whose uterine contractions were not sufficiently inhibited by the betamimetic ritodrine [16]. The results of this study showed that, after magnesium infusion, $90 \%$ of the patients prolonged their pregnancy for more than 48 hours. Interestingly, there were no serious maternal and neonatal side effects [16].

The tocolytic properties of magnesium have been also demonstrated in vitro using both human and rat myometrium [12] [17]. In particular, in non-pregnant human myometrium, magnesium inhibited spontaneous contractions and calcium uptake within minutes, suggesting an extracellular mechanism [17]. However, in primary cultures of pregnant human myometrial cells, more than 20 minutes were required for magnesium to inhibit oxytocin-mediated calcium influx, suggesting that magnesium inhibits myometrial contractility by a combination of extracellular and intracellular mechanisms [18]. Tica and coworkers assessed the effect of magnesium sulfate at different concentrations on spontaneous and oxytocin-induced contractility in myometrial strips, harvested from either pregnant women during cesarean procedures or non-pregnant women during hysterectomy or

Table 1. Changes in primary outcomes of the study.

\begin{tabular}{|c|c|c|c|}
\hline Primary outcomes & $\mathrm{DAV}^{\circledR}(\mathrm{n}=250)$ & Placebo $(n=50)$ & $P$ value \\
\hline Sporadic episodes of preterm uterine contractions & $70(28 \%)$ & $0(0 \%)$ & 0.0001 \\
\hline $\begin{array}{l}\text { Frequent and persistent episodes of preterm } \\
\text { uterine contractions associated with pain }\end{array}$ & $50(20 \%)$ & $30(60 \%)$ & 0.0001 \\
\hline $\begin{array}{l}\text { Maternal need for hospital admission for } \\
\text { threatened preterm labor }\end{array}$ & $50(20 \%)$ & $20(40 \%)$ & 0.001 \\
\hline
\end{tabular}


myomectomy for benign conditions [19]. Although, as expected, the contractions in pregnant and non-pregnant isolated myometrium were of very different magnitude; the data from the two groups were not significantly different. Since oxytocin acts more on pregnant uterus, these results could suggest that magnesium sulfate has a relaxation effect on myometrial cells and not an antagonist action on oxytocin stimulation [19]. This in vitro study suggests efficacy of magnesium sulfate for the treatment or prevention of premature labor, but not for the uterine hyperactivity induced by the active management of labor [19].

Despite several organic and inorganic magnesium salts can be orally administered and are actually used in several food supplements, literature data on the bioavailability of various magnesium forms provide scarce information on the best salt to be used in human supplementation [20]. However, it is well-known that magnesium bioavailability is positively influenced by the concomitant intake of vitamin B6. Indeed, vitamin B-6 and magnesium appear to interact in vivo and it has been suggested that vitamin B-6 may enhance transport or accumulation of magnesium in cells [21].

Uterine contractions are one of the main factors involved in the process leading to preterm delivery. Some clinical and experimental evidence links most preterm births to distinct pathogenic processes which often occur simultaneously and converge on a common biologic pathway characterized by cervical membrane extracellular matrix degradation and myometrial activation, with or without preterm premature rupture of membranes [22].

Both cervical ripening and membrane rupture are mediated by metalloproteases that digest the connective tissue, particularly the collagen, increasing the distensibility of the cervix and the fragility of the membranes [23]. Interestingly, it has recently been shown that ALA inhibits tumor necrosis factor- and thrombin-induced fetal membrane weakening in vitro [24] [25]. Although the mechanism of inhibition of fetal membrane weakening by ALA is still unknown, it has been shown that ALA inhibits NF $\kappa$ B (nuclear factor kappa-light-chain-enhancer of activated $B$ cells), a protein complex that controls the transcription of DNA which is activated by cytokines and thrombin as a part of the induction of matrix metalloproteinase (MMP)-mediated weakening of fetal membranes [26]. Therefore, both the antioxidant and NF $\kappa$ B inhibitory properties of ALA may be involved in such effects.

Considering that the processes of contractions, rupture of membrane and cervical change are strongly linked to each other, it is possible to speculate that a combined treatment of magnesium and ALA may exert several beneficial effects on reducing the risk of preterm delivery.

The present study provides the first direct evidence that a combination of magnesium and ALA may be beneficial to reduce the incidence of premature uterine contractions and related episodes of hospitalization in pregnant women. Although an advantage of this study is the presence of the placebo group, more research is warranted to further confirm the efficacy of this combined supplementation in larger cohorts of patients.

\section{Declaration of Interest}

The authors report no declarations of interest.

\section{References}

[1] Iams, J.D., Newman, R.B., Thom, E.A., Goldenberg, R.L., Mueller-Heubach, E., Moawad, A., et al. (2002) Frequency of Uterine Contractions and the Risk of Spontaneous Preterm Delivery. The New England Journal of Medicine, 346, 250-255. http://dx.doi.org/10.1056/NEJMoa002868

[2] Carson, R.J. (2004) Detection and Prevention of Premature Labour. Neuro Endocrinology Letters, 25, 35-41.

[3] Hubinont, C. and Debieve, F. (2011) Prevention of Preterm Labour: 2011 Update on Tocolysis. Journal of Pregnancy, 2011, 941057. http://dx.doi.org/10.1155/2011/941057

[4] Groom, K.M. (2007) Preterm Labour. Pharmacological Prevention of Prematurity. Best Practice \& Research Clinical Obstetrics \& Gynaecology, 21, 843-856. http://dx.doi.org/10.1016/j.bpobgyn.2007.03.010

[5] Pochwat, B., Szewczyk, B., Sowa-Kucma, M., Siwek, A., Doboszewska, U., Piekoszewski, W., et al. (2013) Antidepressant-Like Activity of Magnesium in the Chronic Mild Stress Model in Rats: Alterations in the NMDA Receptor Subunits. International Journal of Neuropsychopharmacology, 17, 1-13.

[6] Sowa-Kucma, M., Szewczyk, B., Sadlik, K., Piekoszewski, W., Trela, F., Opoka, W., et al. (2013) Zinc, Magnesium and NMDA Receptor Alterations in the Hippocampus of Suicide Victims. Journal of Affective Disorders, 151, 924931. http://dx.doi.org/10.1016/j.jad.2013.08.009

[7] Hall, D.G., Mc Jr., G.H., Corey, E.L. and Thornton Jr., W.N. (1959) The Effects of Magnesium Therapy on the Duration of Labor. American Journal of Obstetrics and Gynecology, 78, 27-32. 
[8] Kurzel, R.B. (1991) Serum Magnesium Levels in Pregnancy and Preterm Labor. American Journal of Perinatology, 8, 119-127. http://dx.doi.org/10.1055/s-2007-999359

[9] Lemancewicz, A., Laudanska, H., Laudanski, T., Karpiuk, A. and Batra, S. (2000) Permeability of Fetal Membranes to Calcium and Magnesium: Possible Role in Preterm Labour. Human Reproduction, 15, 2018-2022. http://dx.doi.org/10.1093/humrep/15.9.2018

[10] Fomin, V.P., Gibbs, S.G., Vanam, R., Morimiya, A. and Hurd, W.W. (2006) Effect of Magnesium Sulfate on Contractile Force and Intracellular Calcium Concentration in Pregnant Human Myometrium. American Journal of Obstetrics and Gynecology, 194, 1384-1390. http://dx.doi.org/10.1016/j.ajog.2005.11.045

[11] Pearson, P.J., Evora, P.R., Seccombe, J.F. and Schaff, H.V. (1998) Hypomagnesemia Inhibits Nitric Oxide Release from Coronary Endothelium: Protective Role of Magnesium Infusion after Cardiac Operations. The Annals of Thoracic Surgery, 65, 967-972. http://dx.doi.org/10.1016/S0003-4975(98)00020-4

[12] Phillippe, M. (1998) Cellular Mechanisms Underlying Magnesium Sulfate Inhibition of Phasic Myometrial Contractions. Biochemical and Biophysical Research Communications, 252, 502-507. http://dx.doi.org/10.1006/bbrc.1998.9680

[13] Hurd, W.W., Natarajan, V., Fischer, J.R., Singh, D.M., Gibbs, S.G. and Fomin, V.P. (2002) Magnesium Sulfate Inhibits the Oxytocin-Induced Production of Inositol 1,4,5-Trisphosphate in Cultured Human Myometrial Cells. American Journal of Obstetrics and Gynecology, 187, 419-424. http://dx.doi.org/10.1067/mob.2002.123897

[14] Raddatz, G. and Bisswanger, H. (1997) Receptor Site and Stereospecifity of Dihydrolipoamide Dehydrogenase for Rand S-Lipoamide: A Molecular Modeling Study. Journal of Biotechnology, 58, 89-100. http://dx.doi.org/10.1016/S0168-1656(97)00135-1

[15] Packer, L., Tritschler, H.J. and Wessel, K. (1997) Neuroprotection by the Metabolic Antioxidant Alpha-Lipoic Acid. Free Radical Biology \& Medicine, 22, 359-378. http://dx.doi.org/10.1016/S0891-5849(96)00269-9

[16] Kawagoe, Y., Sameshima, H., Ikenoue, T., Yasuhi, I. and Kawarabayashi, T. (2011) Magnesium Sulfate as a SecondLine Tocolytic Agent for Preterm Labor: A Randomized Controlled Trial in Kyushu Island. Journal of Pregnancy, 2011, Article ID: 965060. http://dx.doi.org/10.1155/2011/965060

[17] Popper, L.D., Batra, S.C. and Akerlund, M. (1989) The Effect of Magnesium on Calcium Uptake and Contractility in the Human Myometrium. Gynecologic and Obstetric Investigation, 28, 78-81. http://dx.doi.org/10.1159/000293519

[18] Mizuki, J., Tasaka, K., Masumoto, N., Kasahara, K., Miyake, A. and Tanizawa, O. (1993) Magnesium Sulfate Inhibits Oxytocin-Induced Calcium Mobilization in Human Puerperal Myometrial Cells: Possible Involvement of Intracellular Free Magnesium Concentration. American Journal of Obstetrics and Gynecology, 169, 134-139. http://dx.doi.org/10.1016/0002-9378(93)90147-B

[19] Tica, V.I., Tica, A.A., Carlig, V. and Banica, O.S. (2007) Magnesium Ion Inhibits Spontaneous and Induced Contractions of Isolated Uterine Muscle. Gynecological Endocrinology: The Official Journal of the International Society of Gynecological Endocrinology, 23, 368-372.

[20] Coudray, C., Rambeau, M., Feillet-Coudray, C., Gueux, E., Tressol, J.C., Mazur, A., et al. (2005) Study of Magnesium Bioavailability from Ten Organic and Inorganic Mg Salts in Mg-Depleted Rats Using a Stable Isotope Approach. Magnesium Research: Official Organ of the International Society for the Development of Research on Magnesium, 18, 215-223.

[21] Boylan, L.M. and Spallholz, J.E. (1990) In Vitro Evidence for a Relationship between Magnesium and Vitamin B-6. Magnesium Research: Official Organ of the International Society for the Development of Research on Magnesium, 3, 79-85.

[22] Snegovskikh, V., Park, J.S. and Norwitz, E.R. (2006) Endocrinology of Parturition. Endocrinology and Metabolism Clinics of North America, 35, 173-191. http://dx.doi.org/10.1016/j.ecl.2005.09.012

[23] Smith, R., Van Helden, D., Hirst, J., Zakar, T., Read, M., Chan, E.C., Palliser, H., Grammatopoulos, D., Nicholson, R. and Parkington, H.C. (2007) Pathological Interactions with the Timing of Birth and Uterine Activation. Australian \& New Zealand Journal of Obstetrics \& Gynaecology, 47, 430-437. http://dx.doi.org/10.1111/j.1479-828X.2007.00775.x

[24] Moore, R.M., Novak, J.B., Kumar, D., Mansour, J.M., Mercer, B.M. and Moore, J.J. (2009) Alpha-Lipoic Acid Inhibits Tumor Necrosis Factor-Induced Remodeling and Weakening of Human Fetal Membranes. Biology of Reproduction, 80, 781-787. http://dx.doi.org/10.1095/biolreprod.108.073205

[25] Moore, R.M., Schatz, F., Kumar, D., Mercer, B.M., Abdelrahim, A., Rangaswamy, N., et al. (2010) Alpha-Lipoic Acid Inhibits Thrombin-Induced Fetal Membrane Weakening in Vitro. Placenta, 31, 886-892. http://dx.doi.org/10.1016/j.placenta.2010.07.012

[26] Kim, H.S., Kim, H.J., Park, K.G., Kim, Y.N., Kwon, T.K., Park, J.Y., Lee, K.U., Kim, J.G. and Lee, I.K. (2007) $\alpha$-Lipoic Acid Inhibits Matrix Metalloproteinase-9 Expression by Inhibiting NF- $\kappa$ B Transcriptional Activity. Experimental \& Molecular Medicine, 39, 106-113. http://dx.doi.org/10.1038/emm.2007.12 